\title{
La coherencia del conciliacionismo
}

Nicolás Lo Guercio*

Resumen: El artículo se ocupa de la cuestión de los desacuerdos entre pares epistémicos. En primer lugar, se presenta una teoría al respecto que distingue entre desacuerdos fuertes y desacuerdos débiles. Dicha teoría es 'híbrida', en tanto prescribe una reacción conciliadora en un caso (desacuerdos débiles) y una reacción obstinada en el otro (desacuerdos fuertes). En segundo lugar, se presenta una objeción contra las posturas conciliadoras que amenaza la posición aquí defendida. De acuerdo con esta objeción, las posiciones conciliadoras prescriben en algunas circunstancias su propio rechazo. Finalmente, se ofrecen varias razones por las cuales dicha objeción puede ser impugnada, resultando así inocua contra la posición defendida en este artículo.

Palabras clave: epistemología social, desacuerdo, paridad epistémica, conciliacionismo

\section{The coherence of conciliationism}

Abstract: The article discusses peer disagreement. First, an original theory is presented according to which we must distinguish between strong disagreements and weak disagreements. Such a theory is 'hybrid' in the sense that it prescribes a conciliatory reaction in one case (weak disagreements) and a steadfast reaction in the other (strong disagreements). Second, a common objection against conciliatory views is presented which poses a threat against the view above mentioned. According to the objection conciliatory views prescribe in some circumstances its own rejection. Finally, it is argued that this objection can be challenged on several grounds, hence, it represents no danger against the view defended in this article.

${ }^{*}$ Licenciado (2011) y Doctor (2015) en Filosofía por la Universidad de Buenos Aires. Actualmente es becario de posdoctorado del CONICET. Ha dictado clases en la Universidad Nacional de Quilmes, la Universidad Torcuato Di Tella y la Universidad de Buenos Aires. Sus principales áreas de investigación son la epistemología, principalmente la cuestión del desacuerdo, el testimonio y la akrasia epistémica, y la filosofía del lenguaje, especialmente la semántica de los predicados evaluativos y los nombres propios. Dirección electrónica: nicolasloguercio@gmail.com 
Key words: social epistemology, disagreement, epistemic peers, conciliationism

\section{Introducción}

La epistemología del desacuerdo ha adquirido gran notoriedad en los últimos años. Buena parte de los trabajos en este campo se ocupan del desacuerdo entre pares epistémicos, i.e., entre sujetos que poseen aproximadamente la misma evidencia y las mismas virtudes cognitivas. La preocupación principal consiste en determinar en qué medida el descubrimiento del desacuerdo con un par epistémico requiere una revisión doxástica. Las posiciones alrededor de este problema pueden clasificarse, a grandes rasgos, en dos bandos contrapuestos: las posturas conciliadoras mantienen que el descubrimiento del desacuerdo con un par epistémico requiere una revisión doxástica significativa, mientras que las posiciones obstinadas sostienen que en algunos casos es permisible retener la opinión inicial ${ }^{1}$.

Ahora bien, en trabajos anteriores (Lo Guercio 2012, 2015) he defendido una posición propia con respecto a este debate. Dicha postura puede considerarse, en algún sentido, 'híbrida', en tanto distingue dos tipos diferentes de desacuerdos entre pares epistémicos y prescribe una reacción diferente en cada caso (conciliadora en uno, obstinada en el otro). En este artículo me ocuparé de una objeción que, por estar dirigida contra toda posición conciliadora, es potencialmente dañina para la postura antes mencionada.

La objeción en cuestión fue formulada inicialmente por Weatherson y retomada luego por $\mathrm{Elga}^{2}$. He aquí una primera presentación informal de la

1 Para obtener un panorama general del debate, el lector puede consultar las compilaciones de Feldman, R. y Warfield, T., Disagreement, OUP, Oxford, 2010, y Christensen, D. y Lackey, J., The epistemology of disagreement: New essays, Oxford, OUP, 2013.

${ }^{2}$ Cfr. Weatherson, B., "Disagreements, philosophical and otherwise", en Christensen, D. y Lackey, J. (eds), The epistemology of disagreement: New essays, Oxford, OUP, 2013, pp. 54-73. y Elga, A., "How to Disagree about How to Disagree", en Feldman, R. y Warfield, T., Disagreement, Oxford, OUP, 2010, pp. 175-186. Aunque las formulaciones 
misma. Las posiciones conciliadoras mantienen que la reacción doxástica apropiada frente al descubrimiento del desacuerdo con un par epistémico acerca de cierta proposición $P$ es reducir el grado de confianza inicial asignado a $P$. Ahora bien, un tema posible de desacuerdo es la cuestión del desacuerdo entre pares epistémicos. Más aún: es posible desacordar con un par epistémico acerca de esta cuestión. En otras palabras, podemos desacordar con un par epistémico acerca de la posición correcta frente la cuestión del desacuerdo entre pares epistémicos ${ }^{3}$. Esto parece generar prima facie una aporía para el conciliacionismo: si desacuerdo con un par epistémico acerca de la verdad del conciliacionismo y soy conciliacionista parece que debo entonces suspender el juicio acerca del conciliacionismo, o bien reducir significativamente la confianza en el mismo. En palabras de Elga: "las posiciones conciliadoras prescriben en algunos casos su propio rechazo" [Traducción mía] ${ }^{4}$. Esto parece volver a la posición incoherente.

Para ver el punto, supongamos que soy un defensor del conciliacionismo. En virtud de mi compromiso con esta postura debería estar dispuesto a modificar el grado de confianza que asigno a cierta proposición si descubro que desacuerdo sobre la cuestión con alguien a quien considero un par epistémico. Imaginemos ahora que descubro que un colega filósofo, de quien creo justificadamente que es mi par epistémico, defiende una postura obstinada (cree, por lo tanto, que el conciliacionismo es falso). Dados mis compromisos, al enterarme del desacuerdo debería reducir el grado de

mencionadas se circunscriben a la cuestión del desacuerdo entre pares epistémicos, pueden encontrarse formulaciones más generales del problema en Putnam, H., Philosophical Papers: Volume 1. Mathematics, Matter and Method, Cambridge, CUP, 1979; Lewis, D., "Immodest Inductive Methods", Philosophy of Science, 38(1), 1971; Pollock, J. L., "Epistemic norms", Synthese, 71(1), 1987; Field, H., "Apriority as an Evaluative Notion", Boghossian, P. \& Peacocke, C. (eds), New essays on the a priori, Oxford, OUP, 2000, pp. 117-149 y Frances, B., "The refective epistemic renegade", Pbilosophy and Phenomenological Research, 81(2), 2010.

3 Incidentalmente, cabe destacar que esta situación no es meramente posible: la existencia del debate mismo respecto de la epistemología del desacuerdo da cuenta de ello.

${ }^{4}$ Elga, A., "How to Disagree about How to Disagree", en Feldman, R. y Warfield, T., Disagreement, Oxford, OUP, 2010, p. 179. 
confianza que asigno al conciliacionismo y asignar un grado de confianza mayor a las posiciones obstinadas. Si mi posición conciliadora requiere que modifique radicalmente mis creencias entonces parece que si aplico adecuadamente mis propios criterios debo asignar un grado de confianza muy bajo al conciliacionismo, i.e., debo abandonarlo ${ }^{5}$. Así, existen situaciones en la cuales el conciliacionismo recomienda abandonar el conciliacionismo y adoptar una posición obstinada. Esto parece volver a la posición incoherente.

Podría objetarse que no toda posición conciliadora prescribe, en virtud del desacuerdo con un par epistémico, el abandono de la creencia inicial: para un conciliacionista moderado basta con reducir parcialmente la confianza en dicha proposición. Sin embargo, las posiciones moderadas no escapan a la objeción. Supongamos que soy un conciliacionista moderado, i.e., considero que el desacuerdo con un par epistémico requiere una revisión doxástica menor, sin que esto signifique necesariamente abandonar mi creencia original. En ese caso el mero desacuerdo con un par epistémico no será suficiente para que reduzca el grado de confianza en el conciliacionismo tanto como para considerar que lo rechazo. Ahora bien, asumamos que más tarde encuentro un nuevo par epistémico con compromisos obstinados, quien formó su creencia independientemente de la creencia del primero. Si soy fiel al conciliacionismo debo reducir nuevamente mi confianza en el conciliacionismo y aumentar mi confianza en una posición obstinada. Si descubro un número suficiente de pares epistémicos defensores de una posición obstinada y estos formaron sus creencias independientemente, eventualmente deberé reducir tanto el grado de confianza en el conciliacionismo como para rechazarlo, así como aumentar tanto el grado de confianza en las posiciones obstinadas como para aceptarlas. Parece entonces que, en ciertas circunstancias, si soy conciliador, debo rechazar el conciliacionismo. Esto parece indicar que el conciliacionismo es una posición incoherente (al menos en ciertas circunstancias) y eso constituye en principio una razón para abandonarlo.

${ }^{5}$ Esto significa, para varias de las teorías conciliacionistas en la bibliografía, suspender el juicio al respecto. 
En las próximas secciones presentaré esta objeción en la formulación de Elga y discutiré tanto su alcance como algunas soluciones posibles al problema. La estructura del artículo es la siguiente. En primer lugar, presentaré mi propia posición con respecto a los desacuerdos entre pares epistémicos. En segundo lugar, desarrollaré la objeción en detalle. En tercer lugar, examinaré algunas posibles líneas de respuesta. Por último, argumentaré que la objeción no afecta a la teoría defendida en este artículo.

\section{Desacuerdos y perspectiva epistémica}

En esta sección presentaré brevemente la posición desarrollada en trabajos previos acerca de los desacuerdos entre pares epistémicos (Lo Guercio 2012, 2015). Por cuestiones de espacio, lo que sigue no puede más que ser una presentación imperfecta de la posición. No obstante, será suficiente para formular los argumentos que se desarrollarán en las secciones subsiguientes.

El debate acerca de los desacuerdos entre pares epistémicos gira en torno a un problema fundamental, a saber, en qué medida el desacuerdo reconocido con un par epistémico requiere una revisión doxástica. La noción de paridad epistémica, tal como es entendida en la bibliografía, es la de dos individuos tales que ninguno posee una ventaja por sobre el otro en términos de acceso a la evidencia o capacidad para evaluar dicha evidencia:

\section{Paridad epistémica}

Dos sujetos son pares epistémicos respecto de una proposición

$P$ si y solo si (1) poseen (aproximadamente) las mismas virtudes cognitivas, (2) poseen (aproximadamente) la misma evidencia.

Ahora bien, la manera habitual de plantear el problema presupone dos tesis. En primer lugar, la tesis que denominaré, siguiendo a Williamson, Neutralidad Evidencial. Neutralidad Evidencial mantiene que siempre es posible determinar si dos individuos poseen la misma evidencia: "en principio, siempre es posible decidir de manera no controvertida si una proposición constituye evidencia, en el sentido de que una comunidad de investigadores 
puede lograr conocimiento común acerca de si una proposición constituye evidencia para la investigación" [Traducción mía] ${ }^{6}$.

En segundo lugar, el problema presupone que, en principio, siempre es posible determinar de manera no controvertida el grado de apoyo que un cuerpo de evidencia proporciona a cierta proposición. Llamaré a esta tesis Neutralidad Cognitiva. Neutralidad Cognitiva presupone que la relación de apoyo evidencial es diádica: en esta intervienen solamente un cuerpo de evidencia y una proposición. Se deja de lado, en cambio, la posibilidad de que existan diversos criterios subjetivos a la hora de evaluar evidencia o establecer un orden de preferencias entre metas epistémicas, o que estos cumplan un papel a la hora de determinar el grado de apoyo que un cuerpo de evidencia proporciona a una proposición.

En conjunto, Neutralidad Evidencial y Neutralidad Cognitiva implican que la evidencia puede funcionar en todos los casos como árbitro neutral entre actitudes doxásticas en disputa. En otras palabras, la tesis sostiene que siempre es posible, en principio (i.e., haciendo abstracción de los posibles errores de desempeño cognitivo por parte de los agentes), decidir correctamente y de manera no controvertida qué actitud doxástica es la más racional, apelando tan solo a la evidencia. Si esto es correcto, se sigue que todo desacuerdo entre pares epistémicos involucra un error de desempeño cognitivo por parte de alguno de los agentes intervinientes (o de ambos).

Sin embargo, numerosos casos de desacuerdo entre pares epistémicos resultan difíciles de explicar si se suponen estas dos tesis. En relación con la primera de ellas, Neutralidad Evidencial, cabe señalar ciertos desacuerdos particularmente problemáticos, a saber, aquellos que versan sobre la naturaleza misma de la evidencia. Diversas disciplinas albergan desacuerdos respecto de qué cosas son admisibles como evidencia. Estos desacuerdos surgen aun entre individuos bien informados, inteligentes, imparciales, etc., de modo tal que la diferencia de opinión no puede atribuirse ni a la ignorancia ni a la incompetencia de los discordantes. En estos casos no existe una manera no

${ }^{6}$ Williamson, T., The philosophy of philosophy, Oxford, Blackwell, 2007, p. 210. 
controvertida de resolver la cuestión, ni siquiera en principio, apelando solo a la evidencia en tanto árbitro neutral entre opiniones encontradas ${ }^{7}$.

Asimismo, numerosos desacuerdos entre pares epistémicos presuponen diferencias respecto de la naturaleza de la evidencia. Estos desacuerdos también constituyen contra-ejemplos a Neutralidad Evidencial, en tanto no es posible zanjarlos de manera no controvertida apelando solo a la evidencia. Un ejemplo ayudará a ilustrar el punto. Considérese la disputa antropológica examinada por Salmon respecto de la existencia de prácticas caníbales ${ }^{8}$. Una de las cuestiones allí señaladas es que, a pesar de poseer acceso al mismo cuerpo de información, no es claro que los involucrados en la disputa compartan la evidencia. La razón es que mientras que algunos de ellos aceptan como evidencia todos los testimonios que se basan en la observación directa, otros adoptan un estándar más exigente, de acuerdo con el cual solo aquellos testimonios basados en la observación directa por parte de sujetos entrenados cuentan como evidencia. ${ }^{9} \mathrm{De}$ este modo, esta disputa no puede ser zanjada de manera no controvertida apelando solamente a la evidencia como árbitro neutral entre posiciones, como supone la tesis de Neutralidad Evidencial, pues parte de lo que se encuentra en disputa es qué cosas son admisibles como evidencia.

Por otra parte, existen numerosos ejemplos de desacuerdo entre pares epistémicos que resultan problemáticos si se presupone la tesis de Neutralidad Cognitiva. Estos son casos en los que, aun cuando los discordantes acuerden

${ }^{7}$ Cfr. Ibid.

${ }^{8}$ Salmon, M. H., "Art or Science? A Controversy about the Evidence for Cannibalism", en Machamer, P., Pera M. \& Baltas A. (eds.), Scientific Controversies: Philosophical and Historical Perspectives, Oxford, OUP, 2000, pp. 199-212. Los antropólogos mencionados en el artículo de Salmon parecen cumplir con los requisitos para ser considerados pares epistémicos. Aun si no cumplieran con esta condición, sin embargo, es posible imaginar una situación igual en todo a la discutida por Salmon en donde la cumplen.

9 Según esta última posición, demostrar la existencia de prácticas caníbales requiere demostrar la existencia de una práctica institucionalizada y socialmente aprobada de ingesta de carne humana, es decir, la existencia de una costumbre. Observar la ocurrencia de una acción, sin embargo, es diferente de observar una costumbre. Para discernir si cierta acción es la instancia de una costumbre es necesario poseer el entrenamiento adecuado para realizar observaciones de este tipo (básicamente, ser antropólogo). 
respecto de la naturaleza de la evidencia, la disputa no puede resolverse de manera no controvertida apelando a esta, debido a que los sujetos se comprometen con diferentes políticas epistémicas respecto de cómo evaluar la evidencia o bien privilegian metas epistémicas diferentes. Ciertos desacuerdos en filosofía permiten ilustrar el punto. Considérese, por ejemplo, el debate existente en la filosofía analítica respecto del lugar de las intuiciones en el método filosófico ${ }^{10}$. Incluso entre los filósofos que acuerdan en asignar un papel evidencial a las intuiciones existen diferencias respecto del peso que debe otorgarse a esta evidencia: algunos sostienen que la filosofía consiste fundamentalmente en organizar y sistematizar nuestras intuiciones, de manera que el abandono de una intuición solo estaría justificado en virtud de la incorporación de otra de mayor jerarquía ${ }^{11}$; por el contrario, otros filósofos consideran que las intuiciones constituyen el punto de partida del método filosófico pero no existe obligación alguna de honrarlas, sino que siempre es posible abandonarlas en función de compromisos o fines teóricos relevantes ${ }^{12}$. En este caso, a diferencia de lo que ocurría con el desacuerdo antropológico discutido previamente, se presupone un acuerdo básico acerca de qué cosas cuentan como evidencia. En cambio, la disputa concierne a la cuestión de qué peso debe darse a diferentes tipos de evidencia. Si lo dicho hasta aquí es correcto, podría ocurrir que, frente a la misma proposición y la misma evidencia, un filósofo que otorga un peso específico muy grande a las

10 Sobre este tema, véase DePaul, M. y Ramsey, W. (eds) Rethinking intuition: The psychology of intuition and its role in philosophical inquiry, Lanham, Rowman \& Littlefield, 1998, y Pust, J., Intuitions as evidence, New York, Routledge, 2000.

${ }^{11}$ Esta posición fue sostenida por Russell: "Nunca puede haber ninguna razón para rechazar una creencia instintiva a menos que colisione con otras; así, si se encuentra que estas armonizan el sistema completo se vuelve digno de aceptación.” [Traducción mía][Énfasis mío]. Russell, B., The problems of philosophy, Oxford, OUP, 2001, p. 25. Para una defensa más actual de esta idea véase Bealer, G. "Intuition and the Autonomy of Philosophy", en DePaul, M. \& Ramsey, W. (eds), op. cit., pp. 201-240.

${ }^{12}$ Lewis es un ejemplo de esta postura: "Uno arriba a la filosofía ya dotado de un conjunto de opiniones. No es asunto de la filosofía desterrar o justificar en ninguna medida estas opiniones preexistentes, sino tratar de descubrir maneras de expandirlas conformando un sistema ordenado" [Traducción mía] [Énfasis mío]. Lewis, D., Counterfactuals, New York, Routledge, 2013, p. 88. 
intuiciones forme una creencia muy diferente a otro que asigna a estas un papel secundario. Así, existen disputas que presuponen un acuerdo básico respecto de los criterios de admisibilidad para la evidencia, pero no pueden ser zanjadas de manera no controvertida apelando solamente a los criterios para evaluar dicha evidencia, pace Neutralidad Cognitiva.

Incidentalmente, cabe destacar que esta idea se inscribe en una larga tradición dentro de la epistemología y la filosofía de las ciencias, según la cual no existe una única manera objetivamente correcta de sopesar la evidencia, sino que la relación de apoyo evidencial involucra factores subjetivos ineliminables. En otras palabras, la relación de apoyo evidencial es triádica: el grado en que cierto cuerpo de evidencia apoya una proposición es relativo al compromiso con una política epistémica general respecto de cómo evaluar la evidencia ${ }^{13}$.

Otro argumento en favor de esta misma idea hace hincapié en los desacuerdos filosóficos entre sujetos con metas epistémicas diversas. Para ver el punto considérese lo siguiente. Se puede decir que la filosofía es una empresa epistémica. Ahora bien, existe una multitud de dimensiones respecto de las cuales evaluar epistémicamente nuestras creencias y teorías filosóficas. A modo de ejemplo, considérese solo algunas de las metas epistémicas tradicionalmente estimadas como valiosas (además de creer lo verdadero y evitar creer lo falso): éxito predictivo, poder explicativo, parsimonia, simplicidad, elegancia, economía conceptual, claridad conceptual, coherencia, generalidad, adecuación empírica, entre otras. Ahora bien, ocasionalmente, la satisfacción de algunas de estas metas previene la satisfacción de otras. En esos casos qué creer dependerá de tomar una decisión en función de un orden de prioridades. A menudo el grado en que un cuerpo de evidencia apoya una proposición puede variar de acuerdo con el modo en que se construye este orden de prioridades. Supongamos que adoptar cierta creencia vuelve una teoría filosófica más

13 En relación con esta idea véase Douven, I., "Uniqueness revisited", American Philosopbical Quarterly, 46(4), 2009; Kuhn, T. S., "Objectivity, Value Judgment, and Theory Choice", en The essential tension, Chicago, University of Chicago Press, 1977, pp. 320-339; Laudan, L., Science and values: The aims of science and their role in scientific debate, Oakland, University of California Press, 1986 y Machamer, P. K., Pera, M. \& Baltas, A. (eds.), op. cit., entre muchos otros. 
general, pero, en contrapartida, menos simple o parsimoniosa, mientras que rechazarla tiene como consecuencia lo contrario, la teoría resulta más simple pero menos general. Frente a esta decisión, aun cuando compartan la evidencia, distintos sujetos podrían formar actitudes incompatibles si priorizan distintas metas epistémicas. Este tipo de desacuerdos desafía la tesis de Neutralidad Cognitiva, pues no pueden ser zanjados de manera no controvertida apelando a criterios compartidos en relación con estas metas epistémicas.

$\mathrm{Al}$ conjunto de factores hasta aquí discutidos, los denomino perspectiva epistémica básica. Esta incluye, en primer lugar, compromisos acerca de la naturaleza de la evidencia; en segundo lugar, criterios o reglas para evaluar la evidencia; $y$, por último, un orden de preferencias entre metas epistémicas. Así, una vez que se toman en cuenta todos estos factores, parece que: i) la evidencia no puede funcionar en todos los casos como árbitro neutral entre posiciones en disputa, y ii) no todos los desacuerdos entre pares epistémicos involucran un error de desempeño cognitivo o de racionalidad por parte de alguno de los discordantes. En particular, aquellos desacuerdos entre pares epistémicos con perspectivas epistémicas diferentes no pueden ser explicados de esta manera.

Ahora bien, de acuerdo con esta propuesta, las perspectivas epistémicas poseen dos características principales. En primer lugar, algunas perspectivas epistémicas son básicas. Una perspectiva epistémica es básica cuando su racionalidad no puede ser determinada a partir de una perspectiva epistémica diferente de ella misma. En otras palabras, cuando no existe una metaperspectiva desde la cual evaluar su racionalidad ${ }^{14}$. En segundo lugar, las perspectivas epistémicas son inmodestas ${ }^{15}$, es decir, siempre estiman que son más racionales que sus rivales. Como se verá a continuación, estas propiedades están relacionadas.

Para ver el punto considérese lo siguiente. Por un lado, si una perspectiva epistémica básica sancionase que una perspectiva epistémica alternativa e incompatible es más racional, sería incoherente. Para ver el punto

${ }^{14}$ Véase Field, op. cit.

${ }^{15}$ El concepto de perspectiva epistémica inmodesta está inspirado en las ideas de Lewis (op. cit.) en relación con los métodos inductivos, aunque difiere en aspectos importantes. Más adelante discutiré esta idea en detalle. 
imaginemos que cierta perspectiva epistémica básica $A$ prescribe formar la creencia $P$ a la luz de cierta evidencia, pero, a la vez, ordena seguir las recomendaciones de la perspectiva epistémica $B$, la cual, dada la misma evidencia, prescribe creer $\neg P$. En esa situación, la perspectiva epistémica $A$ prescribe actitudes incompatibles: por un lado, recomienda creer $P$ y, por el otro, seguir las recomendaciones de la perspectiva epistémica $B$, la cual prescribe creer $\neg P$. Por este motivo, una perspectiva epistémica básica no puede ordenar acatar las directivas de otra perspectiva epistémica básica diferente e incompatible, so pena de volverse incoherente.

Por otro lado, una perspectiva epistémica básica que evaluase a otra perspectiva epistémica básica incompatible como igualmente racional se volvería arbitraria. Para ver el punto, supongamos que la perspectiva epistémica básica $A$ prescribe creer que $P$ y, a la vez, ordena seguir la perspectiva epistémica básica $B$ (a la cual evalúa como igualmente racional), la cual prescribe creer $\neg P$. En ese caso, el problema no es que la perspectiva epistémica $A$ dé consejos incoherentes, sino que vuelve tanto la creencia en $P$ como en $\neg P$ arbitrarias. Si $A$ prescribe creer que $P$ pero a la vez afirma que $B$, que prescribe creer $\neg P$, es igualmente racional, entonces ¿qué debería creer un agente en esa situación? En este caso, la decisión se vuelve arbitraria: formar una creencia en ese contexto significaría o bien dejar que factores irrelevantes (i.e., factores noepistémicos) jueguen un papel en la decisión o bien arriesgar (esto es, librarse al azar) en favor de una de las posibilidades. Sin embargo, ambas opciones son arbitrarias y, por lo tanto, epistémicamente irracionales ${ }^{16}$.

Ahora bien, intuitivamente, todos estos casos de desacuerdo son relevantes para el tema que nos ocupa, pues todos ellos pueden darse entre pares epistémicos. $\mathrm{Al}$ respecto podría objetarse que dos sujetos con diferentes perspectivas epistémicas no son pares epistémicos. Sin embargo, los desacuerdos en cuestión no pueden explicarse ni por una diferencia en virtudes cognitivas (inteligencia, imparcialidad, atención, etc.) ni por una diferencia en

${ }^{16}$ Este argumento está inspirado en el argumento de White contra el permisivismo. Cfr. White, R., "Epistemic permissiveness", Philosophical perspectives, 19(1), 2005. 
información por parte de alguno de los involucrados (los casos analizados no son casos donde alguno de los involucrados ignore hechos que el otro conoce, sino donde ambos acceden a los mismos hechos pero disienten respecto de su rol evidencial), y estas dos dimensiones son justamente las que resultan relevantes para juzgar la presencia de paridad epistémica. Así, antes que descartar estos desacuerdos conviene incorporarlos al conjunto de fenómenos a explicar e intentar dar cuenta de ellos a partir de una teoría unificada que incorpore las perspectivas epistémicas de los sujetos y permita hilar más fino en cuanto a las nociones de paridad epistémica y los diversos tipos de desacuerdo que puedan surgir, así como respecto de la reacción doxástica requerida por estos en cada caso. Si adoptamos esta postura, tanto Neutralidad Evidencial como Neutralidad Cognitiva (la idea de una relación diádica, completamente objetiva, de apoyo evidencial) deben ser abandonadas.

En trabajos previos sostuve que para dar cuenta de la variedad de desacuerdos antes presentada es necesario diferenciar dos tipos de pares epistémicos, teniendo en cuenta las posibles diferencias en perspectiva epistémica. Así, distinguí entre pares epistémicos fuertes y débiles:

\section{Paridad Epistémica Fuerte}

Dos sujetos son pares epistémicos fuertes respecto de una proposición $P$ si y solo si (1) poseen (aproximadamente) las mismas virtudes epistémicas, (2) poseen (aproximadamente) la misma información relevante para $P$ y (3) sus perspectivas epistémicas son semejantes.

\section{Paridad Epistémica Débil}

Dos sujetos son pares epistémicos débiles respecto de una proposición $P$ si y solo si (1) poseen (aproximadamente) las mismas virtudes epistémicas, (2) poseen (aproximadamente) la misma información relevante para $P$ y (3) sus perspectivas epistémicas son diferentes.

Por el otro lado, las disputas entre distintos tipos de pares epistémicos generan diferentes tipos de desacuerdos: 


\section{Desacuerdo Débil}

Desacuerdos entre pares epistémicos fuertes.

\section{Desacuerdo Fuerte}

Desacuerdos entre pares epistémicos débiles ${ }^{17}$.

De este modo, la pregunta por la reacción doxástica epistémicamente racional frente al descubrimiento del desacuerdo con un par epistémico debe ser respondida en dos partes: por un lado, debemos responder la pregunta por la reacción doxástica racional frente al descubrimiento de un desacuerdo fuerte y, por el otro, es preciso afrontar la pregunta por la reacción doxástica racional frente al descubrimiento de un desacuerdo débil.

La respuesta desarrollada en $(2012,2015)$ a estas dos preguntas debe ser diferente. Por un lado, ante el desacuerdo con un par epistémico fuerte (es decir, ante un desacuerdo débil) debo adoptar una actitud conciliadora. El argumento es el siguiente. Supongamos que desacuerdo con un amigo acerca de cierta proposición $P$. Si mi amigo es un par epistémico fuerte respecto de $P$ este posee aproximadamente la misma evidencia y las mismas virtudes cognitivas que yo, esto es, tiene aproximadamente la misma probabilidad que yo de arribar a una actitud doxástica racional. Dado que además poseemos la misma perspectiva epistémica, al tener noticia del desacuerdo obtengo una razón para modificar mi actitud doxástica inicial respecto de $P$ : después de todo, si desacordamos en estas condiciones, parece que 1) necesariamente alguno de los dos formó una actitud doxástica irracional (o, al menos, menos racional que el otro) y 2) no tengo motivos independientes del desacuerdo para pensar que es más probable que haya sido mi amigo quien se equivocó. Así, el descubrimiento del desacuerdo proporciona en estos casos evidencia de que alguno de los dos (mi par o yo) ha cometido un error al evaluar la información, en el sentido de que no ha adoptado una actitud doxástica racional en virtud de la información que posee y la perspectiva epistémica (compartida) con la cual está comprometido. Dado que no tengo razones para pensar que es más

${ }^{17}$ Se dejan aquí de lado los denominados desacuerdos sin falta (véase MacFarlane, J., Assessment sensitivity: Relative truth and its applications, Oxford, OUP, 2014) y, en general, los desacuerdos acerca de cuestiones evaluativas. 
probable que haya sido mi par quien cometió el error, debo reducir hasta cierto punto la confianza en mi opinión inicial.

Por otro lado, defendí que ante el desacuerdo con un par epistémico débil (es decir, ante un desacuerdo fuerte) no es necesario incurrir en una revisión doxástica significativa. Como se dijo anteriormente, prima facie descubrir el desacuerdo con un par epistémico proporciona evidencia de un error y, por eso, motiva una revisión doxástica. Ahora bien, en los casos de desacuerdo fuerte la diferencia de opinión podría responder a otra razón: la diferencia existente entre sus perspectivas epistémicas. Es decir, el siguiente escenario es posible: i) mi creencia es exactamente la que debería adoptar en virtud de la evidencia que poseo y la perspectiva epistémica con la cual estoy comprometido; ii) la creencia de mi par es exactamente la que él debería adoptar en virtud de la información que posee y su propia perspectiva epistémica y, finalmente iii) la diferencia de opinión se debe a una diferencia de perspectiva epistémica y no a un error de desempeño por parte de alguno de los dos (o ambos).

Este hecho proporciona un derrotador para la evidencia inicial de que cometí un error de desempeño cognitivo. Para ver el punto considérese lo siguiente. Supongamos que observo un objeto y tengo la percepción de que es rojo. Luego descubro que este está iluminado por una luz roja. Esto es evidencia de que el objeto que percibo como rojo podría no serlo: podría verse rojo en virtud de la luz en cuestión. Así, descubrir el dato acerca de la iluminación derrota la evidencia inicial para creer que el objeto es rojo. Análogamente, al descubrir el desacuerdo obtengo evidencia de que cometí un error cognitivo al evaluar la evidencia inicial. Pero luego descubro que la diferencia de opiniones podría no deberse a un error, sino a una diferencia de perspectiva epistémica. Al igual que en el caso anterior, esta información derrota la evidencia de que dicha diferencia se debe a un error de desempeño. Ahora bien, si no tengo evidencia de haber cometido un error de desempeño (o, más bien, esa evidencia fue derrotada), no tengo razones para modificar mi opinión inicial.

Con estas definiciones y criterios en mente, me ocuparé en las siguientes secciones de la cuestión del desacuerdo acerca del desacuerdo y la coherencia 
del conciliacionismo. En última instancia, el objetivo será mostrar que la posición esbozada en esta sección es inmune a dicha objeción.

\section{El conciliacionismo prescribe su propio rechazo}

Me concentraré aquí en la formulación de la objeción desarrollada por $\mathrm{Elga}^{18}$. El argumento puede ser reconstruido del siguiente modo:

1. En algunas circunstancias el conciliacionismo prescribe su propio rechazo.

2. Una posición sobre el desacuerdo entre pares epistémicos que en algunas circunstancias prescribe su propio rechazo es incoherente.

3. Por lo tanto, el conciliacionismo es incoherente.

Elga ofrece una defensa de ambas premisas.

1. En algunas circunstancias el conciliacionismo prescribe su propio rechąo. Asî como descordamos sobre el pronóstico del tiempo, política, deportes y muchas otras cosas, es posible desacordar sobre la respuesta correcta al desacuerdo entre pares epistémicos (esto sucede de hecho entre varios filósofos). Una posición conciliadora debería expedirse sobre estos casos. Pero al hacerlo parece meterse en problemas: supongamos que soy un defensor del conciliacionismo y me encuentro con un par epistémico que mantiene una postura obstinada. Si me tomo en serio el compromiso con el conciliacionismo debería reducir la confianza que asigno al conciliacionismo mismo y aumentar la confianza en una postura obstinada. Si bien el desacuerdo con un solo par podría no ser suficiente para abandonar mi creencia en el conciliacionismo, el desacuerdo con un número suficiente de pares epistémicos cuyas creencias sean independientes me forzará eventualmente a rechazar el conciliacionismo y

${ }^{18}$ Elga, op. cit. La decisión se debe puramente a cuestiones de espacio y comodidad para la exposición. Los argumentos que se desarrollan en las secciones 4 y 5 pueden reformularse en función de la presentación que hace Weatherson. 
adoptar una postura obstinada. Es decir, en ciertas circunstancias el conciliacionismo prescribe su propio rechazo.

2. Una posición sobre el desacuerdo entre pares epistémicos que en algunas circunstancias prescribe su propio rechazo es incoherente. En defensa de esta premisa Elga proporciona un argumento que se puede reconstruir del siguiente modo:

1. Toda posición sobre el desacuerdo entre pares epistémicos es parte de un método inductivo fundamental.

2. Si una posición sobre el desacuerdo entre pares epistémicos prescribe en ciertas circunstancias su propio rechazo, el método inductivo fundamental del que esta forma parte prescribe en ciertas circunstancias su propio rechazo.

3. Si un método inductivo que prescribe su propio rechazo es incoherente, la posición sobre el desacuerdo entre pares epistémicos que forma parte de este, y que prescribe su propio rechazo, es incoherente.

4. Un método inductivo que prescribe su propio rechazo es incoherente.

5. Por lo tanto, una posición sobre el desacuerdo entre pares epistémicos que prescribe su propio rechazo es incoherente.

Veamos qué ofrece Elga, a su vez, en defensa de las premisas de este argumento.

4. Toda posición sobre el desacuerdo entre pares epistémicos es parte de un método inductivo fundamental. Elga no defiende esta premisa. Un método inductivo es un método para evaluar evidencia: ofrece recomendaciones acerca de qué creer en función de un curso de experiencia dado. Sin dudas es plausible que una posición acerca del desacuerdo entre pares epistémicos sea parte de un método inductivo, pues esta ofrece prescripciones acerca del modo de dar cuenta de cierto tipo de evidencia, a saber, la evidencia obtenida al ganar conocimiento del desacuerdo con un par epistémico. Sin embargo, la premisa no solo afirma que una posición sobre el desacuerdo entre pares epistémicos es parte de un método inductivo, sino que mantiene que es parte de un método inductivo fundamental. Como se dijo anteriormente, un método inductivo fundamental es 
un método que no puede ser evaluado a partir de otros métodos, sino que solo puede evaluarse a partir de sí mismo. De este modo, un método inductivo fundamental también ofrece recomendaciones acerca de cuál es el método inductivo que se debe seguir. Esta premisa es crucial para formular la objeción: si las posiciones sobre el desacuerdo entre pares epistémicos no son parte de un método inductivo fundamental el argumento falla (me detendré sobre este punto con más detalle más adelante). Como se dijo, no hay una defensa de esta premisa en el artículo de Elga.

5. Si una posición sobre el desacuerdo entre pares epistémicos prescribe en ciertas circunstancias su propio rechazo, el método inductivo fundamental del cual esta forma parte prescribe, en ciertas circunstancias, su propio rechazo. En defensa de esta premisa Elga ofrece el siguiente argumento:

Más despacio: supongamos que uno tiene la posición $V$ sobre el desacuerdo, y supongamos que uno tiene el método inductivo $M$. La posición $V$ debe ser parte del método inductivo $M$. Entonces, si (dado un curso de experiencia particular) la posición $V$ prescribe el rechazo de la posición $V$, $M$ debe (dado ese curso particular de experiencia) prescribir el rechazo de la posición $V$. Esto se debe a que $M$ prescribe todo lo que prescribe $V$. Pero rechazar la posición $V$ es rechazar $M$, dado que $V$ es una parte de $M$, En consecuencia, $M$ prescribe el rechazo de $M$. Así, si $V$ se auto-socava [it is self-undermining]], entonces $M$ también. [Traducción mía] ${ }^{19}$

6. Si un método inductivo que prescribe su propio rechazo es incoherente, las posiciones sobre el desacuerdo entre pares epistémicos que prescriben su propio rechazo son incoherentes. Elga mantiene que esta premisa se sigue de la anterior:

Ahora supongamos que mi posición acerca del desacuerdo prescribe en ciertas situaciones su propio rechazo. Entonces mi método inductivo también prescribe en ciertas situaciones su propio rechazo. Pues mi posición acerca del desacuerdo es parte de mi método inductivo. Así, para mostrar que las posiciones auto-socavantes acerca del desacuerdo son incoherentes es

${ }^{19}$ Elga, op. cit., nota al pie 6. 
suficiente con probar que los métodos inductivos auto-socavantes son incoberentes. [Traducción mía] [Énfasis mío] ${ }^{20}$

Como argumentaré más adelante, la última afirmación está injustificada. Dejaré esta cuestión en suspenso por el momento para proseguir con la exposición del modo en que Elga motiva cada premisa.

7. Un método inductivo que prescribe su propio rechazo es incoherente. Para justificar esta premisa, Elga se inspira en los trabajos de Lewis y Field ${ }^{21}$. El argumento es el siguiente. Un método inductivo proporciona una regla para responder a distintos cursos de experiencia. Supongamos que el método inductivo $A$ recomienda formar la creencia $X$ frente al curso de experiencia $E$, mientras que el método inductivo $B$ recomienda formar la creencia $Y$ frente al mismo curso de experiencia (y no es posible creer conjuntamente $X$ e $Y$ ). Supongamos además que el método $A$ recomienda seguir el método $B$. En esa situación, el método $A$ da consejos incoherentes: aconseja formar la creencia $X$ frente al curso de experiencia $E$ y a la vez aconseja seguir el método $B$, el cual recomienda formar la creencia $Y$ frente al curso de experiencia $E$. Así, el método $A$ aconseja formar la creencia $X$ frente al curso de experiencia $E$ y no formar la creencia $X$ frente al curso de experiencia $E$ (y formar la creencia $Y$, incompatible con $X$, frente al curso de experiencia $E$ ).

Volviendo al argumento, la conclusión final (8) se sigue entonces por modus ponens a partir de las premisas (6) y (7). Una vez defendida la premisa (2) a partir del argumento que va de (4) a (8), podemos concluir (3) a partir de (1) y (2), también por modus ponens.

\section{Posibles respuestas}

En principio, parece haber al menos dos maneras de solucionar el problema. La primera consiste en abrazar una posición obstinada. En ese caso

\footnotetext{
${ }^{20}$ Elga, op. cit., p. 180.

${ }^{21}$ Lewis, op. cit.; Field, op. cit.
} 
el desacuerdo sobre el desacuerdo lejos está de generar problemas. Para ver el punto, supongamos que mantengo una posición obstinada acerca del desacuerdo entre pares epistémicos. Luego descubro que un par epistémico desacuerda conmigo acerca de la posición correcta frente al desacuerdo con un par epistémico. Mi posición es obstinada, de manera que, frente al desacuerdo con un par epistémico, esta recomienda mantener mi propia posición inicial. Así, parece no haber riesgo de que mi posición prescriba su propio rechazo.

La segunda solución consiste en adoptar una posición conciliadora con alcance restringido. El problema se presenta porque la posición conciliadora considerada recomienda la conciliación sobre absolutamente todo. Si restringimos la norma de manera adecuada el problema desaparece: solo debemos modificar la posición de manera tal que esta requiera ser conciliador ante todo desacuerdo menos ante un desacuerdo sobre el conciliacionismo mismo. De esa manera es posible bloquear la objeción. Una preocupación respecto de esta estrategia, sin embargo, es que resulta a primera vista arbitraria y ad hoc. No parece haber razones, más allá de evitar la inconsistencia, para limitar de este modo la posición.

La solución preferida por Elga es la segunda. Este argumenta que restringir el alcance del conciliacionismo no es en absoluto arbitrario o ad hoc, pues el problema en cuestión no es específico de las posiciones acerca del desacuerdo entre pares epistémicos, sino que aqueja a cualquier método inductivo fundamental (recordemos que para Elga las posiciones sobre el desacuerdo forman parte de un método inductivo fundamental) ${ }^{22}$. Dado que el problema es general, hay razones independientes de la epistemología del desacuerdo para restringir las posiciones conciliadoras de manera tal que estas sean dogmáticas respecto de sí mismas (esto es, que no puedan juzgarse a sí mismas como incorrectas). Para cualquier norma, método o regla fundamental, argumenta Elga, es necesario que esta sea dogmática respecto de sí misma si ha

22 Considérese a modo de ejemplo una regla inductiva fundamental plausible $-\mathrm{y}$, de hecho, defendida por varios filósofos fuera del contexto del debate sobre el desacuerdo entre pares epistémicos- según la cual un sujeto debe formar las creencias que estén mejor apoyadas por la evidencia total que posee en un momento dado. Es posible imaginar una situación donde la evidencia total apoya abandonar esta norma. En ese caso, parece que el método prescribe su propio rechazo. 
de evitar caer en el tipo de incoherencia antes mencionada. Por ende, la restricción del conciliacionismo no es en absoluto arbitraria.

Dejemos de lado por el momento la solución de Elga. Existen varias maneras alternativas de argumentar que la objeción no es exitosa en primer lugar (el problema ni siquiera se plantea), de manera que comprometerse con una solución como la de Elga (o con alguna otra) no es necesario. En primer lugar, se podría argüir que la objeción no es exitosa porque aun cuando el conciliacionismo prescribiese en ciertas circunstancias su propio rechazo eso no mostraría que es falso, sino algo diferente, a saber, que no puede ser racionalmente creído en esas circunstancias. Esto último, sin embargo, no constituye una razón para rechazar el conciliacionismo. En segundo lugar, se puede impugnar la premisa (4) según la cual la posición sobre el desacuerdo entre pares epistémicos es siempre parte de un método fundamental. Por último, podría contra-objetarse que los métodos inductivos fundamentales no pueden revisarse en función de nueva evidencia empírica.

\subsection{Aun si las posiciones conciliadoras prescribieran su propio rechazo, esto no mostraría que son falsas.}

Varios autores señalan que aun si el conciliacionismo, en ciertas circunstancias, fuera una posición auto-socavante [self-undermining], eso no alcanzaría para probar que es falso. La contra-objeción consiste en mostrar principios obviamente verdaderos que, en ciertas circunstancias, se autosocavan. Graves presenta el siguiente ejemplo:

\section{EP1}

Para cualquier sujeto $S$, proposición $P$ y tiempo $T$, si la creencia de $S$ de que $P$ en $T$ es completamente el resultado de una corazonada, una ilusión [wishful thinking], un golpe en la cabeza, una afortunada lesión cerebral o una sacudida producto del rayo láser de un alfa-centauro, entonces la creencia de $S$ de que $P$ en $T$ no está justificada. [Traducción mía] ${ }^{23}$

${ }^{23}$ Graves, S. M., When peers disagree: An essay in the epistemology of peer disagreement, Tesis de Doctorado, New York, University of Rochester, 2010. 
Es claro que EP1 es verdadero. Parece incluso necesariamente verdadero. También es claro, sin embargo, que en ciertas circunstancias EP1 prescribe su propio rechazo. Basta imaginar una situación en la que llego a creer EP1 en base a una corazonada. En la misma línea, Christensen somete a consideración el siguiente principio $^{24}$ :

\section{Humildad Mínima}

Si he pensado casualmente en $P$ por 10 minutos y he decidido que es correcto, y descubro que mucha gente más inteligente que yo y más familiarizada con la evidencia y los argumentos relevantes ha pensado largo tiempo sobre $P$ y de manera independiente, pero unánimemente, ha decidido que $P$ es falsa, debería estar menos confiado en $P$. [Traducción mía ${ }^{25}$

Nuevamente, este principio parece claramente verdadero. Sin embargo, en ciertas circunstancias prescribe su propio rechazo: basta con considerar una situación en la que $P$ es Minimal Humility. De este modo, que un principio prescriba su propio rechazo en ciertas circunstancias no permite concluir que es falso.

Así, la imputación contra el conciliacionismo tiene el problema de sobregeneralizar de un modo indeseable. Para ver el punto basta notar que, si el argumento fuera correcto y esto alcanzara para mostrar la falsedad de un principio epistémico, cualquier posición acerca de la justificación epistémica con un antecedente contingente resultaría falsa. ${ }^{26}$

24 Véase Frances, op. cit. y Matheson, J., "Are Conciliatory Views of Disagreement Self-Defeating?”, Social Epistemology, 2014, pp. 1-15., para otras formulaciones de la misma preocupación.

${ }_{25}$ Christensen, D. "Disagreement as evidence: The epistemology of controversy", Philosophy Compass, 4(5), 2009, p. 763.

${ }^{26}$ Esto es, es posible imaginar un sujeto que cree en el principio confiabilista a través de un proceso no confiable, que cree en el principio evidencialista sin suficiente evidencia, etc. 
La objeción de Elga parece mostrar, en cambio, que un principio epistémico que prescribe en ciertas circunstancias su propio rechazo no puede ser racionalmente creído en esas circunstancias. De eso no se sigue, sin embargo, que el principio sea falso. Tampoco se sigue que otras creencias fundadas en dicho principio no estén justificadas ${ }^{27}$. Podría argumentarse que el hecho de que un principio no pueda ser racionalmente creído en ciertas circunstancias es suficiente para rechazarlo. Sin embargo, esto presenta un inconveniente: si ese fuera el caso deberíamos rechazar cualquier posición sobre la justificación epistémica con un antecedente contingente.

\subsection{Rechazo de la premisa (4)}

La segunda manera de impugnar la objeción consiste en rechazar la idea según la cual las teorías sobre el desacuerdo entre pares epistémicos forman parte de un método inductivo fundamental. Antes de analizar esta estrategia, sin embargo, es necesario realizar una aclaración. Considero que aun si concediéramos la premisa (4) el argumento de Elga fracasaría. Para mostrarlo basta con examinar el paso de (5) a (6). (5) sostiene que si la posición de un sujeto sobre el desacuerdo prescribe su propio rechazo entonces el método inductivo de dicho sujeto también lo hará. La razón es que su posición sobre el desacuerdo es parte de su método inductivo, de manera que todo lo que dice la primera lo dice también el segundo. De esto se sigue que si la posición de un sujeto acerca del desacuerdo es incoherente entonces su método inductivo también lo será, pero no la inversa, esto es, no se sigue que si el método inductivo de un sujeto es incoherente entonces su posición sobre el desacuerdo también lo será. La razón es que un método inductivo dice mucho más que una posición sobre el desacuerdo entre pares, de manera que es posible imaginar un método inductivo incoherente en el sentido relevante (realiza prescripciones incompatibles) que contenga una posición sobre el desacuerdo absolutamente

${ }^{27}$ Para ver el punto considérese lo siguiente: si formo una creencia en el principio confiabilista a través de un proceso no confiable no estoy justificado a creer en el confiabilismo. De eso no se sigue, sin embargo, que no esté justificado en esas mismas circunstancias en creer que el objeto que tengo delante mío es una computadora, si formé esta creencia a través de un proceso confiable. 
coherente. Para ver el punto basta con imaginar un sujeto cuyo método inductivo contiene el principio confiabilista más una posición obstinada acerca del desacuerdo entre pares epistémicos. Dicho método inductivo prescribe en ciertas circunstancias su propio rechazo: en una circunstancia en la que el sujeto forma su creencia en el confiabilismo a través de un proceso de formación de creencias poco confiable el confiabilismo prescribe el rechazo del confiabilismo, es decir, realiza prescripciones incoherentes. Dado que la tesis confiabilista es parte de su método inductivo (este último dice todo lo que dice aquella), este realiza prescripciones incoherentes. Sin embargo, de esto no se sigue que su posición sobre el desacuerdo realice prescripciones incompatibles. Como argumenté anteriormente, para las posiciones obstinadas los casos de desacuerdo sobre desacuerdos no resultan problemáticos. En consecuencia, aun cuando un método inductivo que prescribe su propio rechazo fuera incoherente, de esto no sigue que la posición sobre el desacuerdo entre pares que aquél contiene también lo sea.

Una manera caritativa de reformular la objeción es considerar a las posiciones acerca del desacuerdo entre pares epistémicos como métodos inductivos fundamentales, y no solamente como parte de un método inductivo fundamental. Si se considera al conciliacionismo como un método inductivo fundamental en sí mismo entonces las consideraciones del párrafo anterior se vuelven irrelevantes y la objeción atendible. Como dije anteriormente, los métodos inductivos fundamentales son reglas para asimilar evidencia, con la particularidad de que no se puede apelar a una regla diferente de ellos mismos para juzgar si el método es correcto. En ese caso, frente al desacuerdo sobre el desacuerdo el conciliacionismo prescribe su propio rechazo y la aceptación de una posición diferente, de manera que proporciona consejos incoherentes.

Sin embargo, el artículo de Elga carece de una defensa de la idea de que una posición acerca del desacuerdo entre pares epistémicos constituya un método inductivo fundamental. Esto representa un problema, pues el siguiente escenario parece plausible. Un sujeto cree en el conciliacionismo. Sin embargo, no evalúa la corrección del conciliacionismo de acuerdo con el conciliacionismo mismo, sino que posee un método inductivo más fundamental que utiliza para ese fin. Ese método inductivo determina que el sujeto debe creer en el conciliacionismo salvo en las circunstancias en que este proporcione consejos incoherentes. En este último caso, el método inductivo 
fundamental prescribe abandonar el conciliacionismo así como todas las creencias fundadas en este, y adoptar la regla alternativa que goce de mayor apoyo. En ciertas circunstancias, e.g., cuando el sujeto desacuerda con un par epistémico acerca de la respuesta correcta frente al desacuerdo con un par epistémico, el conciliacionismo proporciona consejos incoherentes. Por lo tanto, en esas circunstancias el sujeto debe rechazar el conciliacionismo y las creencias que formó en base a este y adoptar la posición alternativa. Así, la prescripción del método inductivo fundamental no es incompatible con ninguna de las prescripciones del conciliacionismo, pues el primero tiene prioridad sobre el segundo. Por supuesto, si el compromiso con el conciliacionismo es fundamental no es posible juzgar la corrección del conciliacionismo a partir de un método diferente, pero Elga nos debe un argumento de por qué no podría haber un método inductivo más fundamental que el conciliacionismo que determinase, en ciertas circunstancias, que es necesario rechazarlo. Así, si el conciliacionismo no es un método inductivo fundamental el problema no se presenta, de manera que la objeción de Elga se diluye y su solución, por tanto, resulta innecesaria.

\subsection{Los métodos inductivos fundamentales no prescriben su propio rechazo}

Existe una tercera línea de respuesta posible. Esta concede que el conciliacionismo es un método inductivo fundamental pero niega que los métodos inductivos fundamentales prescriban su propio rechazo. Esta línea de respuesta puede extraerse del trabajo de Field -aunque este se ocupa de la objeción en toda su generalidad, no de la cuestión del conciliacionismo en particular ${ }^{28}$. En su presentación de la objeción que nos ocupa Elga menciona que su argumento se apoya en una formulación anterior de Field en el artículo antes citado. Sin embargo, en ese trabajo Field argumenta que el problema [puzzle] es en última instancia inocuo. Veamos por qué.

${ }^{28}$ Field, op. cit. 
Field considera que la pregunta principal a contestar es si un método inductivo fundamental puede ser visto como empíricamente vulnerable [empirically defeasible] o, por el contrario, debe ser visto como empíricamente inmune. El autor plantea el problema de manera general. La primera parte dice así. Queremos que nuestros métodos inductivos sean confiables (en el presente y en el futuro). Podemos investigar empíricamente, a su vez, si nuestro método inductivo fue confiable en el pasado. Es posible que el resultado de esta investigación sea negativo, esto es, es posible que lleguemos a la conclusión de que nuestro método fue poco confiable en el pasado, y que algún otro método fue más confiable. Dado que asumimos que la confiabilidad del método en el pasado es un indicador de la confiabilidad del método en el futuro, podemos concluir que nuestro método será menos confiable que el alternativo en el futuro. En ese caso, parece razonable pensar que deberíamos cambiar de método. Todo lo dicho anteriormente, afirma Field, se aplica a métodos nobásicos, pero también a métodos básicos o fundamentales.

La segunda parte del problema afirma que la conclusión de la primera parte es incoherente. Si mi método implica que, en virtud de la evidencia empírica $E$, no siga el método (y siga en cambio otro método), entonces proporciona instrucciones inconsistentes. Así, la conclusión parece ser que un método fundamental empíricamente vulnerable es inconsistente ${ }^{29}$.

$\mathrm{El}$ asunto, de acuerdo con Field, es que la primera parte del problema contiene un error. El error consiste en pensar que si un método inductivo básico fue poco confiable en el pasado lo será en el futuro. Esto es un error porque los métodos inductivos básicos se 'auto-corrigen': al obtener evidencia empírica de su falta de confiabilidad pasada lo que hace el método es asimilarla y tenerla en cuenta a la hora de determinar sus aplicaciones futuras. Así, las nuevas aplicaciones del método no serán iguales a las anteriores. En otras palabras: un método inductivo es un método que prescribe ciertas creencias en virtud de cierta evidencia. Si el método inductivo prescribía formar la creencia $P$ en un grado $C$ en virtud de la evidencia $E$ y luego se obtiene evidencia

${ }^{29} \mathrm{El}$ artículo de Field busca defender justamente la idea de que los métodos inductivos fundamentales son a priori, en el sentido de que son empíricamente inmunes (no son revisables en virtud de evidencia empírica). 
empírica contra la confiabilidad del método mismo, entonces la próxima vez que se encuentre con la evidencia $E$ el método tendrá en cuenta la evidencia de su poca confiabilidad pasada y ya no prescribirá el mismo grado de creencia en $P$, sino uno diferente. La evidencia acumulada va modificando las aplicaciones futuras del método. La evidencia empírica de la falta de confiabilidad pasada de cierto método inductivo fundamental, de este modo, no constituye evidencia contra el método, sino que es incorporada por este, afectando así sus aplicaciones futuras ${ }^{30}$.

Así, Field concluye que los métodos inductivos fundamentales son inmunes a la evidencia empírica, esto es, si un método es básico para mí no me es posible siquiera reconocer cierta pieza de evidencia empírica como evidencia contra el método. Para ver nuevamente el punto, considérese el siguiente ejemplo. Supongamos que utilizo la regla perceptiva "Si ves algo rojo, cree que es rojo" y esta es para mí una regla fundamental. Luego encuentro evidencia empírica contra su confiabilidad, a saber, observo que, bajo ciertas condiciones de iluminación, objetos que no son rojos se ven rojos. ¿Qué debo hacer con la regla? Parece razonable mantener que la regla (fundamental) fue descartada o refutada. Sin embargo, afirma Field, esto es un error. Si la regla es sensible a evidencia que va más allá de la apariencia de los objetos (en particular, es sensible a evidencia respecto de la iluminación de los objetos) entonces esta no era fundamental después de todo; era operativa una regla más fundamental (una meta-regla) que estipulaba que debo creer que algo es rojo si lo veo rojo, a menos que la percepción se dé en circunstancias en las cuales la regla es poco confiable. Por otra parte, si la regla realmente fuera fundamental no queda claro por qué debería modificarla en virtud de la evidencia respecto de la luz (si la regla fuera realmente fundamental no sería sensible a ese tipo de evidencia). Así, por un lado, si la regla inicial era básica entonces la evidencia sobre la luz no la socava [undermines] sino que se incorpora a esta y es tomada en cuenta en sus futuras aplicaciones; por otro lado, si la evidencia sobre la luz realmente lleva a modificar la regla, entonces esta no era realmente fundamental (pues de otro modo no sería sensible a un tipo de evidencia que no está contemplada en la regla misma).

${ }^{30}$ Véase Field, op. cit., p. 133. 
Es fácil ver cómo puede utilizarse la idea de Field para contestar la objeción de Elga. Elga argumenta que una posición sobre el desacuerdo es (parte de) un método inductivo fundamental. Cuando un conciliacionista descubre que un par epistémico desacuerda respecto de la verdad del conciliacionismo, este obtiene evidencia de la falta de confiabilidad pasada del conciliacionismo mismo. Ahora bien, si Field está en lo correcto, la situación abre dos posibilidades. Por un lado, el conciliacionista podría modificar su método. En ese caso, el conciliacionismo no podría considerarse un método fundamental, pues es claro que una regla más básica está siendo usada para modificar la regla conciliacionista, una meta-regla que mantiene que hay que ser conciliador a menos que haya desacuerdo con un par epistémico respecto del conciliacionismo. Ahora bien, si el conciliacionismo realmente es un método inductivo fundamental lo que ocurre es que el método incorpora la evidencia de la falta de confiabilidad pasada provista por el desacuerdo y esta afecta la aplicación futura del método mismo. En ese caso, sin embargo, ni el conciliacionista se ve obligado a abandonar su postura ni el método ofrece prescripciones incompatibles, de manera que la objeción queda desarticulada.

Podría objetarse que esta última respuesta a la objeción es en realidad muy similar a la solución que propicia el propio Elga. Argumentar que los métodos inductivos fundamentales son empíricamente inmunes es bastante parecido a mantener que son dogmáticos. En definitiva, lo que sucede en ambos casos es que un método, si es fundamental, no puede declararse a sí mismo incorrecto. Sin embargo, este parecido no es más que superficial. Lo que mantiene Field no es que los métodos inductivos son dogmáticos, sino que ni siquiera pueden reconocer cierta pieza de evidencia empírica como evidencia que podría socavarlos. Esto es sensiblemente diferente de la posición mantenida por Elga, según la cual un método fundamental es sensible a evidencia empírica en su contra, pero debe ignorarla o desestimarla, con el sencillo objetivo de preservar la consistencia. Así, los métodos inductivos fundamentales son dogmáticos para Elga porque desestiman la evidencia empírica que los socava, mientras que son empiricamente inmunes para Field porque no son siquiera sensibles a dicha evidencia.

Hasta aquí me he ocupado del problema formulado por Elga. Argumenté que (i) aun cuando el conciliacionismo prescribiese en ciertas circunstancias su propio rechazo eso no mostraría que es falso, ni 
proporcionaría una razón para rechazarlo; (ii) Elga nos debe una defensa/motivación de la premisa que mantiene que una posición sobre el desacuerdo epistémico forma parte de (o es) un método inductivo fundamental; y (iii) aun cuando esta premisa fuese cierta es posible argumentar que la objeción fracasa a partir de la idea de que los métodos inductivos fundamentales son empíricamente inmunes.

\section{Una posición coherente}

Sin dudas la cuestión de la coherencia de las posiciones conciliadoras reviste interés para todos aquellos dedicados a la cuestión del desacuerdo entre pares epistémicos. Sin embargo, la motivación principal para discutir la cuestión en este artículo consiste en que la objeción presentada es potencialmente dañina para la posición defendida en la sección segunda. La teoría allí defendida es parcialmente conciliadora en tanto requiere, al menos para ciertos desacuerdos (desacuerdos débiles), reducir el grado de confianza en la propia creencia. Ahora bien, dado que la objeción en cuestión afecta a las posiciones conciliadoras, vale la pena examinar si impacta negativamente en la teoría defendida en las secciones anteriores. En lo que sigue, argumentaré que la posición defendida en este artículo es inmune a la objeción de incoherencia que, presuntamente, afecta a las posiciones conciliadoras.

La objeción formulada por Elga, dicho brevemente, es que las posiciones conciliadoras ofrecen recomendaciones inconsistentes en escenarios donde está en juego el desacuerdo con un par epistémico acerca de la posición correcta ante el desacuerdo con un par epistémico. De este modo, la objeción parece amenazar en principio a todas aquellas posiciones que recomiendan una actitud conciliadora ante esta situación. Para averiguar si la posición defendida en este artículo consigue evadir el problema conviene entonces examinar cuál es su recomendación ante el desacuerdo con un par epistémico acerca de la actitud correcta frente el desacuerdo con un par epistémico.

La posición que defendí en esta tesis mantiene que el desacuerdo entre pares epistémicos débiles, que denominé desacuerdo fuerte, prescribe una reacción obstinada, mientras que el desacuerdo entre pares epistémicos fuertes, 
que denominé desacuerdo débil, requiere una actitud conciliadora. La pregunta que se presenta es ¿en qué categoría debería situarse al desacuerdo acerca del desacuerdo? ¿es este un desacuerdo fuerte o uno débil? Si el desacuerdo sobre el desacuerdo es un tipo de desacuerdo fuerte la objeción es bloqueada desde el inicio, pues en esos casos la teoría prescribe una reacción obstinada. Si el desacuerdo sobre el desacuerdo es un tipo de desacuerdo débil, en cambio, la objeción parece prima facie problemática, pues la teoría prescribe en esos casos una actitud conciliadora.

La objeción, tal como es presentada por Elga, presupone que una posición sobre el desacuerdo entre pares epistémicos es parte de un método inductivo fundamental. Esto es mencionado explícitamente por Elga. Ahora bien, si una posición sobre el desacuerdo entre pares epistémicos es parte de un método inductivo fundamental entonces esta forma parte de lo que he denominado la perspectiva epistémica básica de un sujeto: por un lado, determina el modo de responder a un curso de experiencia dado y, por el otro, es tal que su estatus no puede juzgarse respecto de ningún otro método más allá de sí mismo. Si la posición sobre el desacuerdo entre pares epistémicos es parte de un método inductivo fundamental y este es a su vez parte de una perspectiva epistémica, entonces la posición sobre el desacuerdo entre pares epistémicos es parte de una perspectiva epistémica. El desacuerdo sobre el desacuerdo, por lo tanto, debe ser considerado un caso de desacuerdo fuerte, esto es, un caso de desacuerdo entre sujetos que a pesar de ser pares epistémicos poseen perspectivas epistémicas significativamente diferentes. En los casos de desacuerdo fuerte, recuérdese, la teoría defendida en este artículo recomienda ser obstinado. Como se dijo anteriormente, si la teoría recomienda, ante el desacuerdo sobre el desacuerdo, una reacción obstinada, el problema no se presenta siquiera.

$\mathrm{Si}$, por el contrario, las posiciones sobre el descuerdo entre pares epistémicos no forman parte de un método inductivo fundamental, la teoría permanece del mismo modo inmune a la objeción. Podría mantenerse que las posiciones sobre el desacuerdo entre pares epistémicos no forman parte de un método inductivo fundamental, de manera que es posible que dos pares epistémicos desacuerden sobre el desacuerdo y, a la vez, compartan suficientemente su perspectiva epistémica. Así, los desacuerdos sobre desacuerdos serían desacuerdos débiles. En esos casos, la teoría prescribe una 
actitud conciliadora. Si esto es correcto, parece que mi posición prescribe en esas circunstancias una disminución de la confianza en la teoría misma. Este argumento contiene un error, sin embargo. El mismo presupone que se utiliza la teoría sobre la que se desacuerda para resolver el desacuerdo mismo. Sin embargo, eso solo ocurriría si las teorías sobre el desacuerdo fuesen fundamentales (es decir, si no pudiesen juzgarse más que a partir de sí mismas). Pero en el escenario propuesto las teorías sobre el desacuerdo no son fundamentales, de manera que el desacuerdo sobre el desacuerdo no se resolverá apelando a la teoría que está en disputa sino a un método inductivo de un nivel superior, una meta-regla. Así, aun si se considera que los desacuerdos sobre desacuerdos son desacuerdos débiles, la teoría defendida en esta tesis logra evadir la objeción de incoherencia.

\section{Conclusión}

En este artículo he presentado una posición propia respecto del desacuerdo entre pares epistémicos. Dicha posición es 'híbrida', en tanto recomienda una reacción conciliadora frente a ciertos tipos de desacuerdo y una posición obstinada frente a otros. Asimismo, he discutido una objeción comúnmente esgrimida en contra las posiciones conciliadoras acerca del desacuerdo entre pares epistémicos. De acuerdo con dicha objeción, en ciertas circunstancias el conciliacionismo prescribe su propio rechazo. Esas circunstancias son aquellas en las cuales un defensor de la posición conciliadora desacuerda con un par epistémico acerca de la posición correcta respecto del desacuerdo entre pares epistémicos. Si esto es correcto, dice la objeción, el conciliacionismo es incoherente y, por lo tanto, debe ser abandonado. El problema es que la objeción amenaza la posición antes mencionada, en tanto esta es parcialmente conciliadora. Por este motivo, examiné en la segunda mitad del artículo el alcance y la plausibilidad del argumento.

La discusión mostró que la objeción puede ser impugnada por varias razones. En primer lugar, aun si su conclusión fuera verdadera, esto no mostraría que el conciliacionismo es falso; solo permitiría concluir que en ciertas circunstancias no puede ser creído racionalmente. Por otra parte, si la objeción fuera exitosa probaría demasiado: si se rechazara toda teoría que 
prescribe en ciertas circunstancias su propio rechazo, deberíamos rechazar toda teoría acerca de la justificación epistémica con un antecedente contingente. En segundo lugar, el argumento se basa en una premisa controvertida que no está argumentada, a saber, que toda posición acerca del desacuerdo entre pares epistémicos es parte de un método inductivo fundamental. En tercer lugar, argumenté que aun si se concede dicha premisa, el carácter empíricamente inmune de los métodos inductivos permite bloquear la conclusión del argumento. Finalmente, mostré que la objeción es inocua en relación con la teoría híbrida antes mencionada.

\section{Bibliografía}

Bealer, George,"Intuition and the Autonomy of Philosophy." en DePaul, Michael, y Ramsey, William (eds.), Rethinking intuition: The psychology of intuition and its role in philosophical inquiry, Lanham, Rowman \& Littlefield, 1998, pp. 201 240.

Christensen, David, "Disagreement as evidence: The epistemology of controversy", Philosophy Compass, 4(5), 2009, pp. 756-767.

Christensen, David y Lackey, Jennifer, The epistemology of disagreement: New essays. Oxford, Oxford University Press, 2013

DePaul, Michael y Ramsey, William (eds.), Retbinking intuition: The psychology of intuition and its role in philosophical inquiry, Lanham, Rowman \& Littlefield, 1998.

Douven, Igor, "Uniqueness revisited." American Philosophical Quarterly, 46(4), 2009, pp. 347-361.

Elga, Adam, "How to Disagree about How to Disagree", en Feldman, Richard, y Warfield, Ted (eds.), Disagreement, Oxford, Oxford University Press, 2010, pp. 175-186.

Feldman, Richard, y Wareld, Ted, Disagreement, Oxford, Oxford University Press, 2010. 
Field, Hartry, "Apriority as an Evaluative Notion", en Boghossian, Paul y Peacocke, Christopher (eds.), New essays on the a priori, Oxford, Oxford University Press, 2000, pp. 117-149.

Frances, Bryan, "The reflective epistemic renegade", Philosophy and Phenomenological Research, 81(2), 2010, pp. 419-463.

Graves, Shawn M., When peers disagree: An essay in the epistemology of peer disagreement. Tesis de doctorado, University of Rochester, 2010.

Kuhn, Thomas S., “Objectivity, Value Judgment, and Theory Choice.”, en The essential tension, Chicago, University of Chicago Press, 1997, pp. 320-339.

Laudan, Larry, Science and values: The aims of science and their role in scientific debate, Berkeley, University of California Press, 1986.

Lewis, David, "Immodest Inductive Methods", Philosophy of Science, 38(1), 1971, pp. 54-63.

Lewis, David, Counterfactuals, New York, Routledge, 2013

Lo Guercio, Nicolás, "Philosophical peer disagreement", Logos \& Episteme, 3(3), 2012, pp. 459-467.

Lo Guercio, Nicolás, Desacuerdos entre pares epistémicos: problemas y soluciones. Tesis doctoral, Universidad de Buenos Aires, 2015.

MacFarlane, John, Assessment sensitivity: Relative truth and its applications, Oxford, Oxford University Press, 2014.

Machamer, Peter K., Pera, Marcello, \& Baltas, Aristides (eds.), Scientic controversies: philosophical and historical perspectives, Oxford, Oxford University Press, 2000.

Matheson, Jonathan, "Are Conciliatory Views of Disagreement SelfDefeating?”, Social Epistemology, 29(2), 2014, pp. 145-159.

Pollock, John L., “Epistemic norms”, Synthese, 71(1), 1987, pp. 61-95.

Pust, Joel, Intuitions as evidence, New York, Routledge, 2000.

Putnam, Hilary, Philosophical Papers: Volume 1, Mathematics, Matter and Method, Cambridge, Cambridge University Press, 1979. 
Russell, Bertrand, The problems of philosophy, Oxford, Oxford University Press, 2001.

Salmon, Merrilee H., "Art or Science? A Controversy about the Evidence for Cannibalism", en Machamer, Peter, Pera, Marcello y Baltas, Aristides (eds.), Scientic Controversies: Philosophical and Historical Perspectives, Oxford, Oxford University Press, 2000, pp. 199-212.

Weatherson, Brian, "Disagreements, philosophical and otherwise", en Christensen, David y Lackey, Jennifer (eds.), The epistemology of disagreement: New essays, Oxford, Oxford University Press, 2013, pp. 54-73.

White, Roger, "Epistemic permissiveness", Philosophical perspectives, 19(1), 2005, pp. 445-459.

Williamson, Timothy, The philosophy of philosophy, New York, Blackwell Publishing, 2007.

Recibido: 05/2017; aceptado: 10/2017 